\title{
Measurement of spectacle lenses: A review
}

\section{Medición de lentes oftálmicas: Una revisión}

\author{
Paloma Guadalupe Mendoza-Villegas $\left(1,{ }^{*}\right)$, Gerardo Trujillo-Schiaffino $(1, \mathrm{~A})$, \\ Didia Patricia Salas-Peimbert(1,A), Marcelino Anguiano-Morales(1), \\ Daniel Malacara-Doblado(2,A), Luis Francisco Corral-Martínez ${ }^{(1)}$
}

1. División de Estudios de Posgrado e Investigación, Instituto Tecnológico de Chihuahua, Ave. Tecnológico 2909, 31310 Chihuahua, México.

2. División de Óptica, Centro de Investigaciones en Óptica, Lomas del Bosque 115, 37150 León, México.

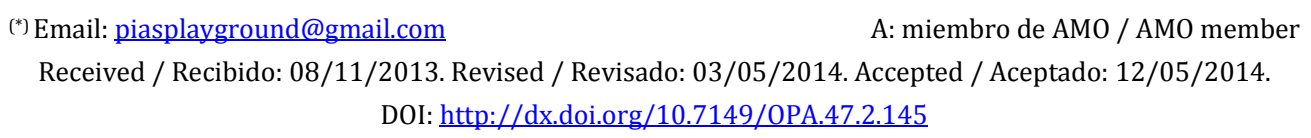

\begin{abstract}
:
Spectacle lenses are the most commonly used solution for refractive errors of the eye, such as myopia, hyperopia, presbyopia and astigmatism. To correct a refractive error, the spectacle lens needs to have the correct lens power and an appropriate distribution of power along the surface of the lens, to measure these characteristics several methods have been developed, this paper presents a review of some of these methods and their optical principles.
\end{abstract}

Key words: Spectacle Lens, Lens Power, Lensometers.

\section{RESUMEN:}

Las lentes oftálmicas son la solución más utilizada para la corrección de errores refractivos del ojo, tales como miopía, hipermetropía, presbicia y astigmatismo. Para corregir adecuadamente un error refractivo, la lente especular debe tener la potencia correcta, así como una adecuada distribución de potencia a lo largo de la lente. Para medir dichas características se han desarrollado diversos métodos, este artículo presenta un resumen de algunos de las técnicas más relevantes así como los principios ópticos que utilizan.

Palabras clave: Lente Oftálmica, Potencia de Lentes, Lensómetros.

\section{REFERENCES AND LINKS / REFERENCIAS Y ENLACES}

[1]. International Council for Education of People with Visual Impairment. http://www.icevi.org/ICEVI General Information Spanish.html.

[2]. D. Atchison, G. Smith, Optics of the Human Eye, Buttherworth-Heinemann, Edinburgh (2000).

[3]. J. Schwiegerling, Field guide to Visual and Ophthalmic Optics, SPIE Press, Bellingham (2004). DOI

[4]. The Vision Care Center: https://es.vsp.com/enes/cms/edc/glossary.html.

[5]. D. Meister, "The Optics of Free-Form Progressive Lenses", 20/20 Mag., 131-134 (2008).

[6]. F. Z. Fang, X. D. Zhang, A. Weckenmann, G. X. Zhang, C. Evans, "Manufacturing and measurement of freeform optics", CIRP Ann.-Manuf. Techn. 62, 823-846 (2013). DOI

[7]. Basic Optical Laboratories. Free-Form: The principles. http://www.basicoptical.co.za/files/Freeform\%20THE\%20PRINCIPLES.pdf

[8]. C. Fowler, P. Latham, Spectacle Lenses: Theory and Practice, Butterworth-Heinemann, Oxford, pp. 68, (2001).

[9]. W.E.Humphrey, "Lensmeter utilizing non parallel light”, US Patent App 4,182,572 (1980). 
[10]. W. E. Humphrey, "Lensometer with guided lens movement for progressive addition lens", US Patent App 5,303,022 (1994).

[11]. K. Gnanvo, Z. Yan, J. Bougrenet, L. Liu, "Large aperture automatic focimeter for the measurement of optical power and other optical characteristics of ophthalmic lenses", Appl. Opt. 41, 5997-6005 (2002). DOI

[12]. D. Malacara, M. Servín, Z. Malacara, Interferogram Analysis for Optical Testing, Marcel Dekker, New York (1998).

[13]. J. C. Wyant, F. D. Smith, "Interferometer for measuring power distribution of ophthalmic lenses", Appl. Opt. 14, 1607-1612 (1975). DOI

[14].F. Granados-Agustín, A. Jaramillo-Núñez, D. Sacramento-Solano, M. Percino-Zacarias, A. CornejoRodríguez, "Lensmeter using a lateral shearing interferometer", Optik 123, 1425-1428 (2012). DOI

[15]. H. Medecki, E. Tejnil, J. Goldberg, K. A. Bokor, "Phase shifting point diffraction interferometer", Opt. Lett. 21, 1526-1528 (1996). DOI

[16]. E. Acosta, S. Chamadoira, R. Blendowske, "Modified point diffraction interferometer for inspection and evaluation of ophthalmic components", J. Opt. Soc. Am. A 23, 623-627 (2006). DOI

[17].C. Koliopoulos, O. Kwon, R. Shagam, J. C. Wyant, C. R. Hayslett, "Infrared point diffraction interferometer", Opt. Lett. 3, 118-120 (1978). DOI

[18]. B. Vohnsen, S. Castillo, D. Rativa, "Wavefront sensing with axicon", Opt. Lett. 36, 846-848 (2011). D0I

[19]. P. Goodwin, J. Wyant, Field Guide to Interferometric Optical Testing, SPIE, Washington (2006). DOI

[20].C. Illueca, C. Vazquez, C. Hernandez, V. Viqueira, "The use of Newton rings for characterising ophthalmic lenses", Ophthal. Physiol. Opt. 18, 360-371 (1998). DOI

[21]. D. Malacara, Optical Shop Testing, Wiley, New Jersey (2007). DOI

[22]. E. Pateras, "An interferometric method for measuring the dioptric and prismatic power of ophthalmic lenses”, e-J. Sci. Technol. 3, 41-48 (2010).

[23]. Y. Nakano, K. Murata, "Talbot interferometry for measuring the focal length of a lens", Appl. Opt. 24, 3162-3166 (1985). DOI

[24]. L. Bernardo, O. Soares, "Evaluation of the focal distance of a lens by Talbot interferometry", Appl. Opt. 27, 296-301 (1988).

[25]. J. C. Bhattacharya, A. K. Aggarwal, "Measurement of the focal length of a collimating lens using the Talbot effect and the moiré technique", Appl. Opt. 30, 4479-4480 (1990). DOI

[26]. K. V. Sriram, M. P. Kothiyal, R. S. Sirohi, "Direct determination of focal length by using Talbot interferometry", Appl. Opt. 31, 5984-5987 (1992). DOI

[27]. D. Malacara-Doblado, "Measuring the curvature of spherical wavefronts with Talbot interferometry", Opt. Eng. 36, 2016-2024 (1997). DOI

[28]. O. Kafri, I. Glatt, The Physics of Moiré Metrology, Wiley, New York (1989).

[29]. J. A. Quiroga, D. Crespo, E. Bernabeu, “Fourier transform method for automatic processing of Moiré deflectograms", Opt. Eng. 38, 974-982 (1999). DOI

[30]. I. Glatt, O. Kafri, "Determination of the focal length of nonparaxial lenses by moiré deflectometry", Appl. Opt. 26, 2507-2508 (1987). DOI

[31]. E. Keren, K. Kreske, O. Kafri, "Universal method for determining the focal length of optical systems by moiré deflectometry", Appl. Opt. 27, 1383-1385 (1988). DOI

[32]. J. Vargas, J. Gómez-Pedrero, J. Alonso, J. Quiroga, “Deflectometric method for the measurement of user power for ophthalmic lenses", Appl. Opt. 49, 5125-5132 (2010). DOI

[33]. H. Canabal, J. A. Quiroga, E. Bernabeu, "Automatic processing in moiré deflectometry by local fringe direction calculation", Appl. Opt. 37, 5894-5901 (1998). DOI

[34].X. Wei, T. V. Heugten, L. Thibos. "Validation of a Hartmann-Moiré wavefront sensor with large dynamic range", Opt. Express 17, 14180-14185 (2009). DOI

[35]. D. Salas-Peimbert, G. Trujillo-Schiaffino, J. Gonzalez-Silva, S. Almazán-Cuellar, D. Malacara-Doblado, "Simple Hartmann test data interpretation for ophthalmic lenses", Rev. Sci. Instrum. 77, 043102 (2006). DOI 
[36].C. Castellini, F. Francini, B. Tiribilli, "Hartmann test modification for measuring ophthalmic progressive lenses", Appl. Opt. 33, 4120-4124 (1994). DOI

[37]. C. Zhou, W. Wang, K. Yang, X. Chai, Q. Ren, "Measurement and comparison of the optical performance of an ophthalmic lens based on a Hartmann-Shack wavefront sensor in real viewing conditions", Appl. Opt. 47, 6434-6441 (2008). DOI

[38].W. Doyle, "Robust automated instrument for measuring the focal length of a lens", Opt. Eng. 51, 43601-43601 (2012). DOI

[39]. E. A. Villegas, P. Artal, "Spatially resolved wavefront aberrations of ophthalmic progressive power lenses in normal viewing conditions", Optom. Vis. Sci. 80, 106-114 (2003). DOI

[40]. L.-L. Qin, J.-C. Yu, "Research on Hartmann test for progressive addition lenses", Proc. SPIE 7283, 728318 (2009). DOI

[41]. C. Gonzalez, E. R. Villegas, L. Carretero, A. Fimia, "Ronchi test for testing the powers of bifocal intraocular lenses”, Ophthal. Physl. Opt. 17, 161-163 (1997). DOI

[42]. L. Carretero, C. Gonzalez, A. Fimia, I. Pascual, "Application of the Ronchi test to intraocular lenses: A comparison of theoretical an measured results", Appl. Opt. 32, 4132-4137 (1993). DOI

[43]. P. Buah-Bassuah, B. Tiribilli, M. Vassalli, G. Molesini, "A simple portable lens meter", Appl. Opt. 42, 5086-5090 (2003). DOI

[44]. T. Bothe, W. Li, C. Kopylow, W. Jüptner, "High resolution 3D shape measurement on specular surfaces by fringe reflection", Proc. SPIE 5457, 411-422 (2004). DOI

\section{Introduction}

Nowadays around 124 million people around the world present ocular errors [1] affecting their everyday life due to poor vision quality. To correct conditions such as myopia, hyperopia, astigmatism and presbyopia there is a simple inexpensive solution, to wear spectacle lenses.

But is important to consider that the spectacle lens must be adequate to the subject using it, otherwise is more a problem than a solution. Wearing glasses with the wrong prescription has as consequences nausea, headaches, blurred vision, strained eyes, dizziness, vertigo and of course low visual acuity.

Due to the importance of ophthalmic lenses to correct visual problems, a correct measure of the characteristics of the lens is necessary. There are three principal entities involved in a lens prescription, the wearer, the optician and the manufacturer. For all of them correct lens measurement is essential. In the case of the manufacturer power measurements are used for quality control and for the optician to make sure is giving the right prescription to the patient.

Manufacturers often will use lens mappers or contact profilometry to test their lenses since they must provide a lens with the best quality all over its surface. On the other hand opticians often recur to lensmeters that measure optical power in the vertex of the lens, which is convenient in the measurement of single vision lenses. Ophthalmic lenses have aberrations in the periphery. However, most people using single vision lenses sees trough the center of the lens and turns their head to move the visual point instead of using peripheral vision.

Trough the years several methods and techniques have been developed to test spectacle lenses. Some of them just for experimental purposes and a few are the base for the commercial lensmeters available these days.

In this article a review of techniques used mainly for lens power measurements is presented. Many of this techniques can be applied in a wide field, but we will focus in measurement of power in specular lenses.

\section{Refractive errors of the eye}

The eye is a complex optic system which function consists in form an image and sends it to our brain; the quality of such an image will depend on the incident light in to the eye. Figure 1 shows the most important optical structure of the human eye concerning image formation. 


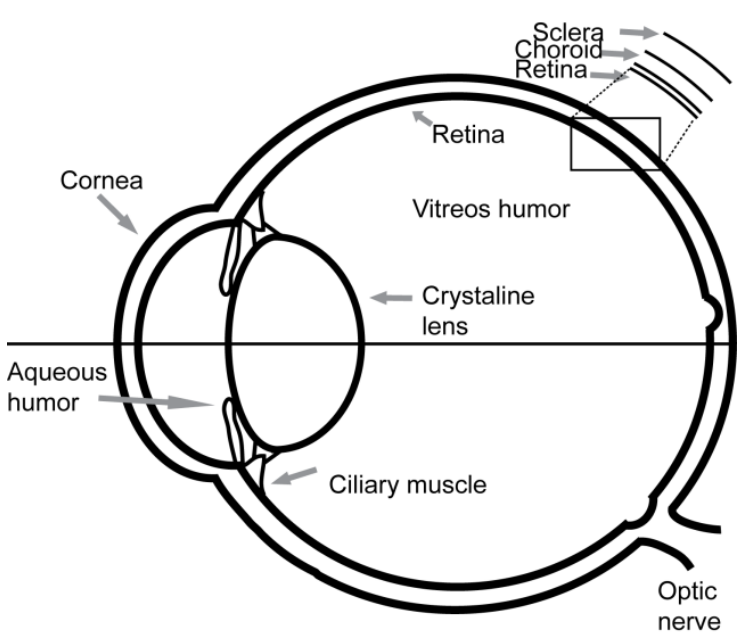

Fig. 1 General case of formation of the retinal image and image forming beams.

Image forming light leaves the observed object enters the eye through the cornea, and is refracted by the cornea and the lens to be focused at the retina.

Apparently image formation is simple, however many factors affect vision quality. For example, if corneal surface is too irregular or its curvature is not adequate image will be distorted. Another example occurs when the lens is not capable to alter its shape enough to focus the image of an observed object [2].

Ideally when the eye is fixated in an interest object, image is focused at the retina. Nonetheless image can be focused at the retina only if is inside the range of accommodation of the eye.

Emmetropia is a state of refraction where a point at an infinite distance from the eye is conjugate to the retina. An emmetropic eye is capable of providing the eye a range of accommodation to focus objects at near and far distance (see Figs. 2(a) and 2(b)).

Ametropia is a state where refractive error is present [3]. An ocular error is also known as a refractive error of the eye. Below there is a brief description of some of the most common refractive errors.

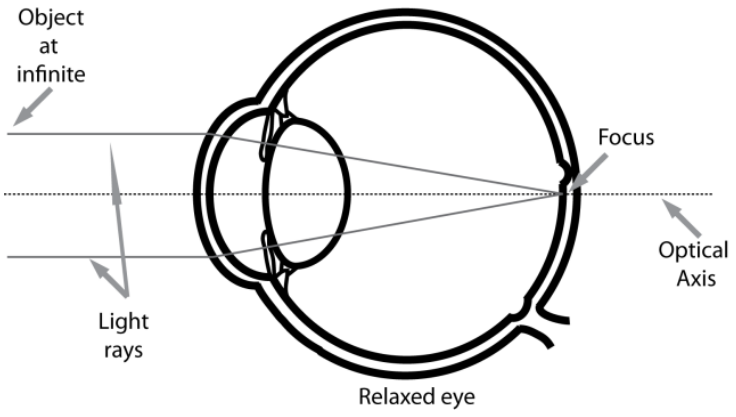

(a)

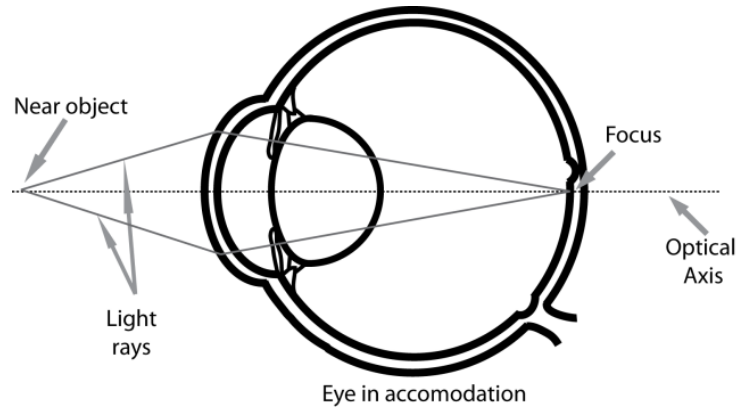

(b)

Fig. 2. Emmetropic eye focusing an object (a) at infinite, (b) at near point.

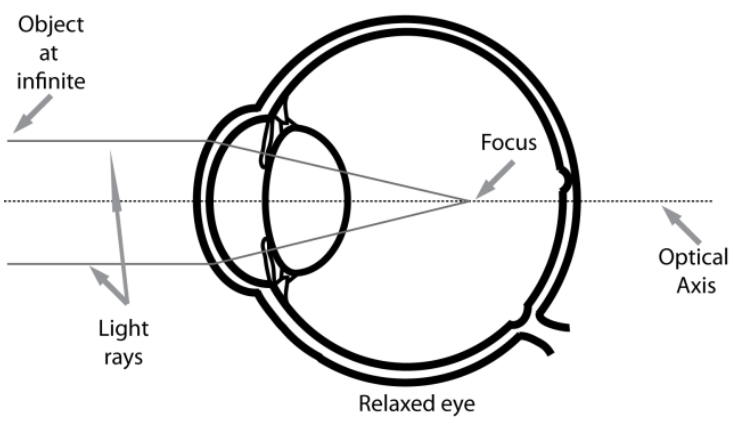

(a)

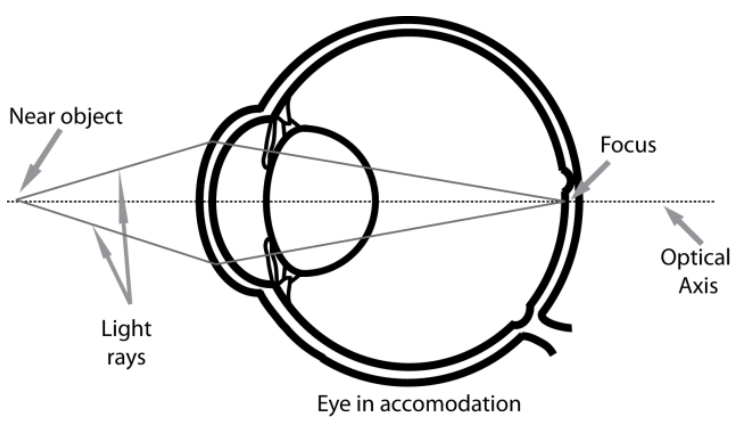

(b)

Fig. 3 Myopic eye focusing an object (a) at infinite, (b) at emmetropic near point. 


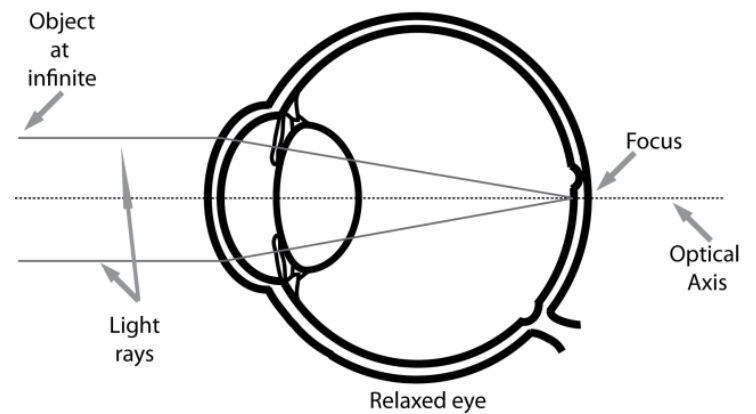

(a)

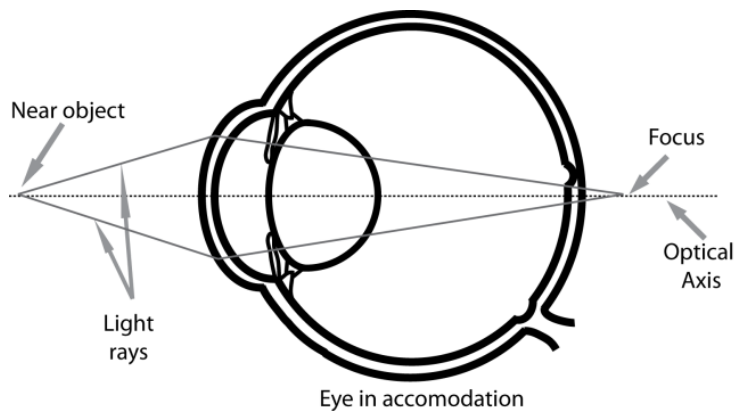

(b)

Fig. 4. Hyperopic eye focusing an object (a) at infinite, (b) at emmetropic near point.

\section{2.a. Myopia}

In the myopia, also called near-sightedness, the eye is too long or the lens has too high power. The subject won't be able to focus far objects (Fig. 3).

\section{2.b. Hyperopia}

In hyperopia, also called far-sightedness, the lens' power is too weak or the eyeball is too short. The subject won't be able to focus near objects (Fig. 4).

\section{2.c.Presbyopia}

Presbyopia is the progressive loss of the amplitude of accommodation with age. The subject won't be able to focus near objects (Fig. 5).

\section{2.d. Astigmatism}

In astigmatism, the refractive error changes with meridian, due to an irregular surface of the cornea, lens or the eye itself (Fig. 6). It causes mild blurry vision and sometimes eyestrain [4].

All refractive errors of the eye can be corrected using ophthalmic lenses.

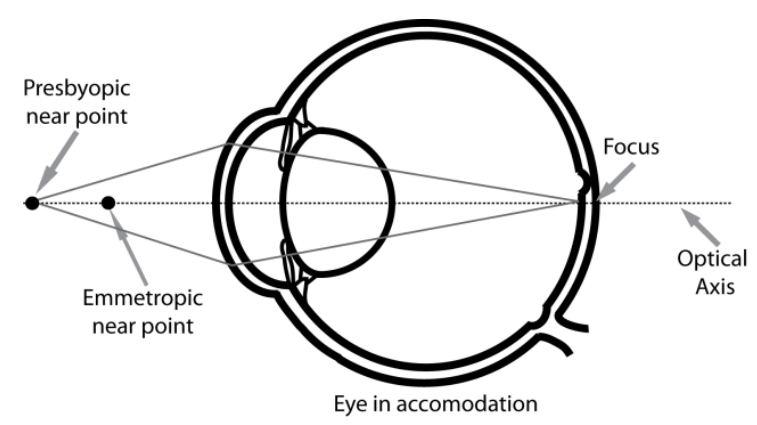

Fig. 5. Image formation: Presbyopia.

\section{Ophthalmic lenses}

The principal refractive components of the human eye are the lens and the cornea. By wearing an ophthalmic lens to correct a refractive error we are adding a new refractive component to the vision system. This way the eye supported by the lens power provided for the ophthalmic lens is now able to focus objects forming quality images at the retina.

This paper will discuss only types of spectacle lenses, leaving contact lenses aside.

\section{3.a. Single vision lenses}

Consist of two surfaces that provide uniform optical power. The eye is mobile being able to look through different portions of the lens, that's the reason why off-axis aberrations are important in designing spectacle lenses. Spherical or sphero-cylindrical single vision lenses are prescribed for people with myopia, hyperopia or astigmatism (see Figs. 7 and 8), even presbyopes that use two separate lenses for far and near vision.

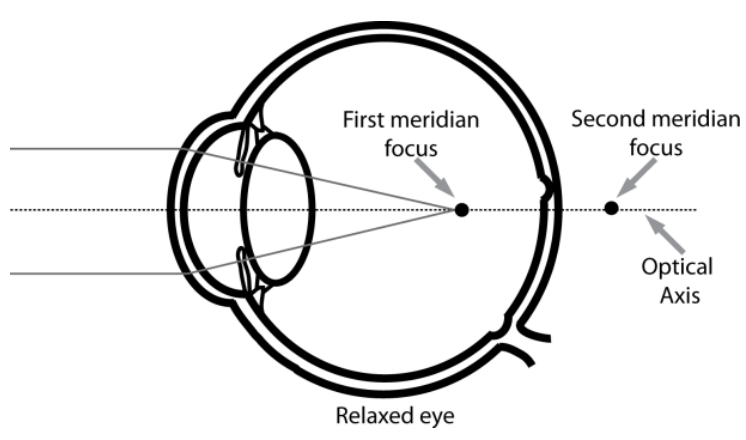

Fig. 6. Image formation: Astigmatism. 


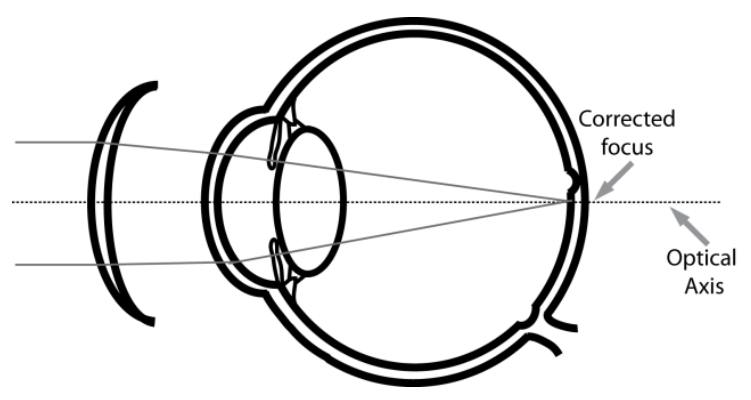

(a)

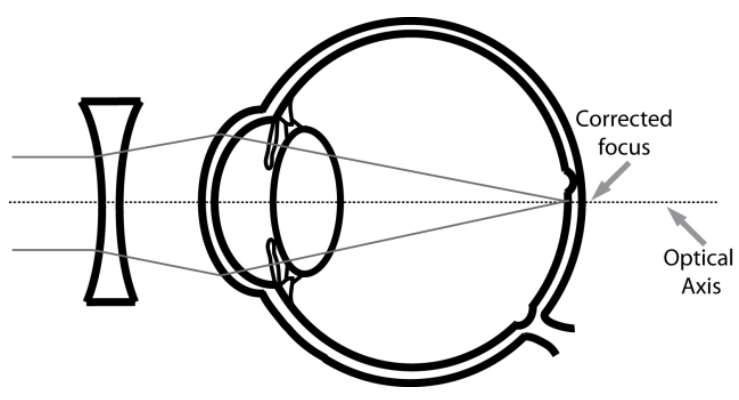

(b)

Fig. 7. Single vision lenses: (a) Negative to lens used to correct myopia, (b) Positive lens used to correct hyperopia.

Cylindrical lenses are used to correct simple astigmatism, in which the refraction in one principal meridian of the eye is myopic or hyperopic and the other meridian is emmetropic. The lens has no refracting power in one of the two principal meridians.

Toric lenses are required to correct compound astigmatism, in which the refraction in both major meridians of the eye is myopic or hyperopic. The lens has refracting power in both principal meridians.

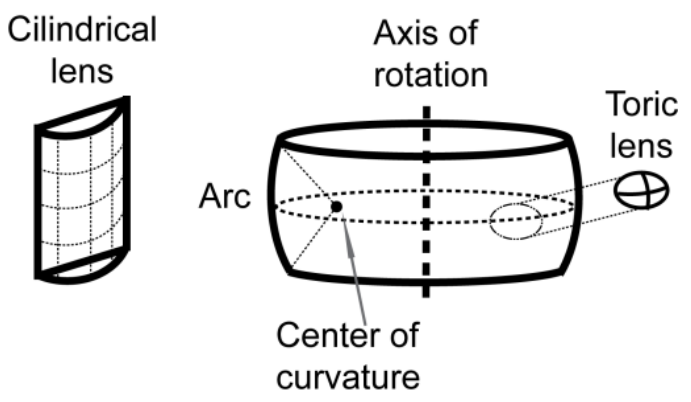

Fig. 8. Cylindrical and toric lenses.

\section{3.b. Multifocal lenses}

These types of lenses provide two or more focal distances. For example a person with myopia develops presbyopia with age, would need one lens with the correct power to see far objects (myopia), and another set of glasses to see near objects (presbyopia). A bifocal lens would correct both problems since the lens has two different curvatures fused in one lens to combine the distance portion (main lens), and the reading zone (segment). There are also the trifocals that combine a third vision zone to see medium distance objects. Bifocals and trifocal lenses cause the subject who wears it a sense of image jump when they go from one focal zone to another. Progressive addition lenses are an alternative to bifocal and trifocal lenses. These lenses are designed to progressively change focal power from upper to lower zones; this provides a continuous vision at all distances without an image jump.

Recently freeform lenses are becoming a more suitable solution for the wearer of ophthalmic lenses, since they can be fabricated for the specific needs of the individual [5]. Free form lenses are irregular surfaces without a rotational axis, modeled with CAD software [6]. The precision of free form lenses is of $0.01 \mathrm{D}$, also they have a reduced power error in all the field of view and power is specified point by point. Another advantage of this lenses is that the consider user power. User power is the one perceived by the wearer when looking at any portion of the lens, it depends on the surface of the lens, position an orientation respect to the eye [7].

\section{Methods to test ophthalmic lenses}

Most lensmeters measure vertex power in ophthalmic lenses and have tools or special procedures that allows them to measure PPL's, in the case of free form lenses nanometric accuracy is required and user power is measured, for this lenses contact and non contact techniques are available for measuring. Contact methods require large amounts of time and can damage the surface. Non-contact methods like interferometric techniques are accurate and fast but sensitive to environmental conditions such as dust, oil, heat, etc [6]. 
In this section several techniques will be discussed, mainly measurement of vertex power with non contact procedures will be addressed.

\section{4.a. Focimeter}

Focimeter [8] is an optical device capable of measuring the power of an ophthalmic lens. The device consists of an illuminated target, a collimating lens, a reticle and an eyepiece. The ophthalmic lens to test has to be placed at the focal point of the spectacle lens. Light emerging from the lens enters an eyepiece with an internal reticle. The user must move the target axially until it is simultaneously in focus with the reticle. This situation only occurs when the light from the ophthalmic lens is collimated. The target position $d$ is related to the power of the spectacle lens $(P)$ by:

$$
P=\frac{d-f}{f^{2}},
$$

This focimeter is useful to measure spherical, cylindrical and prismatic power in ophthalmic lenses, as shown in Fig. 9 is used to test single vision lenses. Humphrey $[9,10]$ modified the focimeter to use it with non-parallel light and later used it to measure progressive power lenses, by adding a computer indicated lens movement protocol (see Fig. 10). The source of light impinges in four discrete lens surfaces to obtain 4 beams, sphero-cylindrical power and prismatic power can be obtained in the point equidistant to the beams.

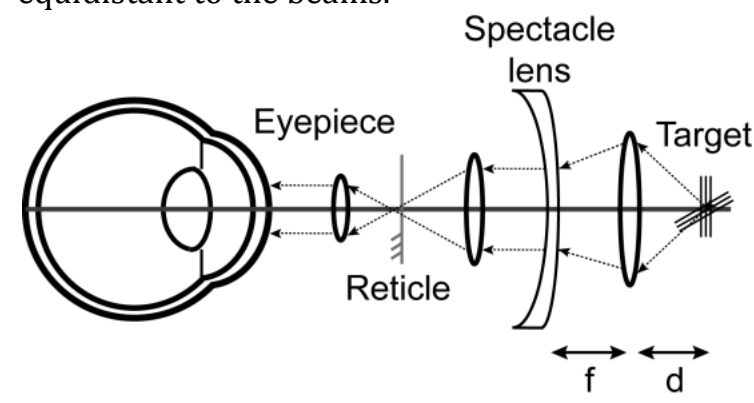

Fig. 9. Focimeter.

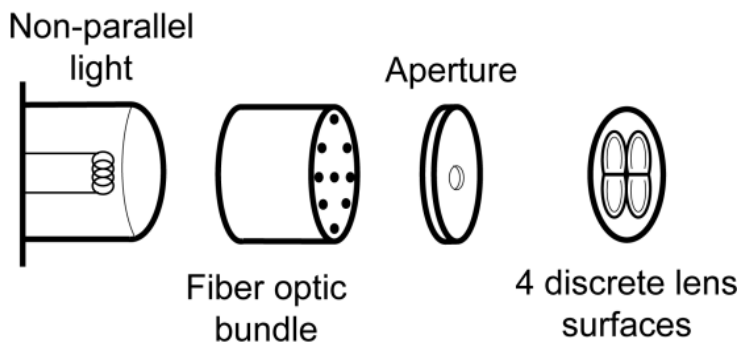

Gnanvo et al [11] presented a variation of the focimeter; they used a position sensitive detector to receive the beam of light emerging from the spectacle lens (see Fig. 11). To measure all the surface of the lens there is a system to hold and move the lens so that the beam of light passes through all the area. This variation allows measuring progressive power lenses.

\section{4.b. Lateral shear interferometer}

An interferometer is an instrument that combines two coherent beams of light to produce an interference pattern. This pattern will depend on the intensities and phases of the beams; according to the principle of superposition the resulting amplitude of the disturbance is the sum of amplitudes of the interfering beams. [12]

The lateral shear interferometer is the result of two sheared wavefronts so it doesn't require a reference wavefront (see Fig. 12).

There are several methods to build a lateral shear interferometer [13] one of the simplest consists of a laser beam going through a microscope objective and a collimating lens; light leaving the lens impinges on the front and back surfaces of a plane parallel plate (Fig. 12). The plate surfaces reflect the laser beam and produce a displacement $(S)$ according to:

$$
\frac{S}{t}=\sin 2 i \sqrt{N^{2}-\sin ^{2} i},
$$

where $t$ is the plate thickness, $N$ is the refraction index and $i$ is the incidence angle of light.

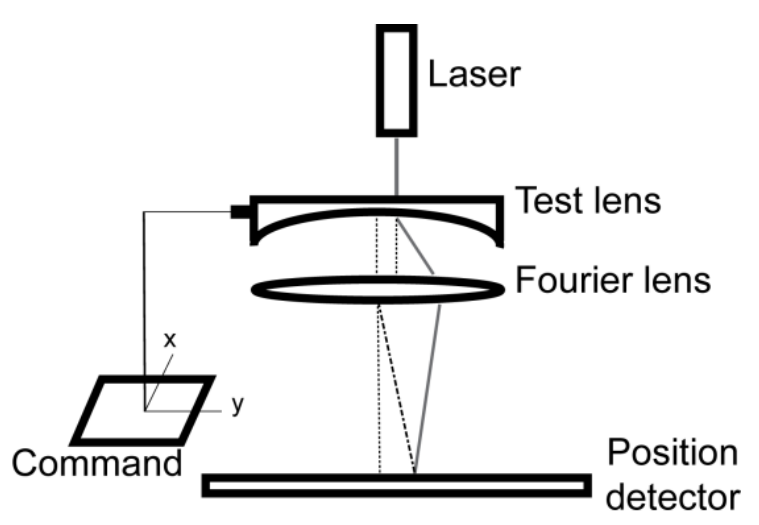

Fig. 11. Focimeter used by Gnanvo et al [11].

Fig. 10. Non parallel source light used by Humphrey [10]. 


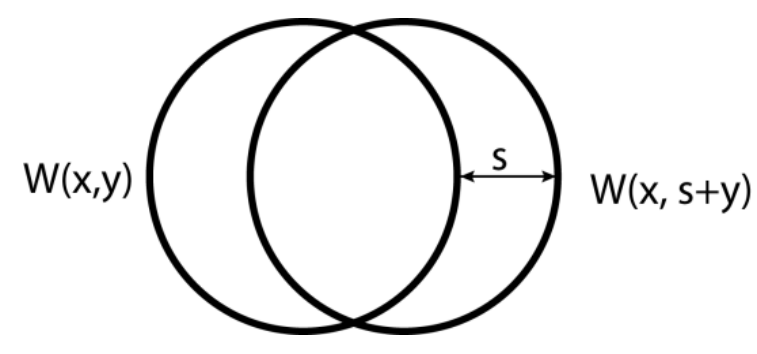

Fig. 12. Two sheared wavefronts.

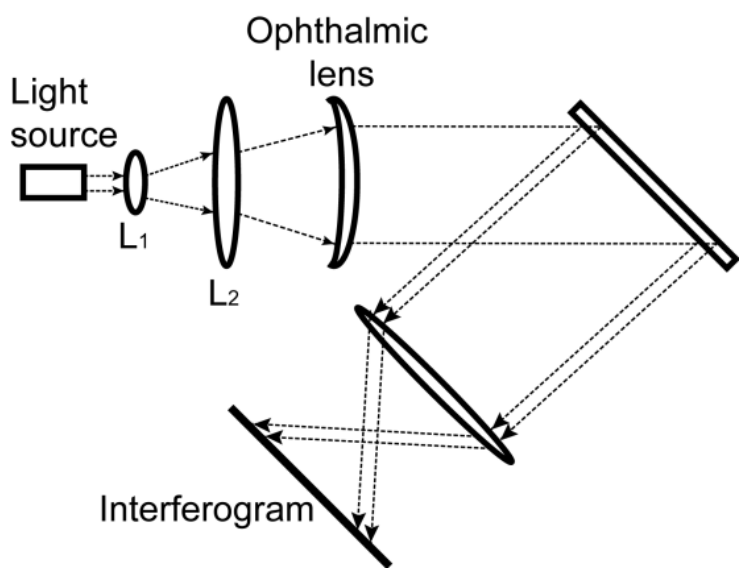

Fig. 13. Lateral shear interferometer

The lens to be tested must be placed in a converging or diverging beam of light, this way the light emerging from the lens is collimated. Collimated light hits a plane parallel plate, which reflects part of the beam from the front surface and the other part from the back surface. The reflected beams are laterally sheared a distance $S$. If the beam leaving the lens is not collimated, interference fringes will appear in the common area of the two reflected beams. The spacing of the fringes is inversely proportional to the local power of the lens. The light leaving the lens is either converging to, or diverging from, a point a distance $L$ from the lens. The separation $x$ of the fringes is given by:

$$
x=\frac{\lambda L}{S} .
$$

The local power of the lens is given by:

$$
P=\frac{1}{L}=\frac{\lambda}{x S} .
$$

Wyant and Smith [13] presented a lateral shear interferometer to measure ophthalmic lenses; the system is shown in Fig. 14. According to their

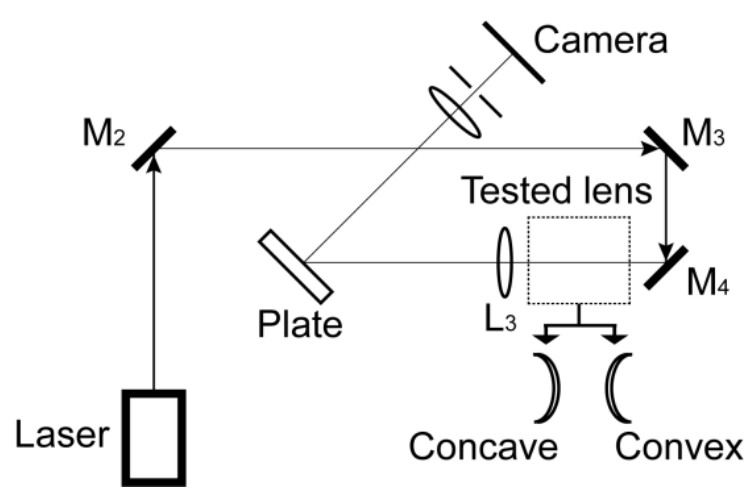

Fig. 14. Lateral shear interferometer proposed by Wyant and Smith [13].

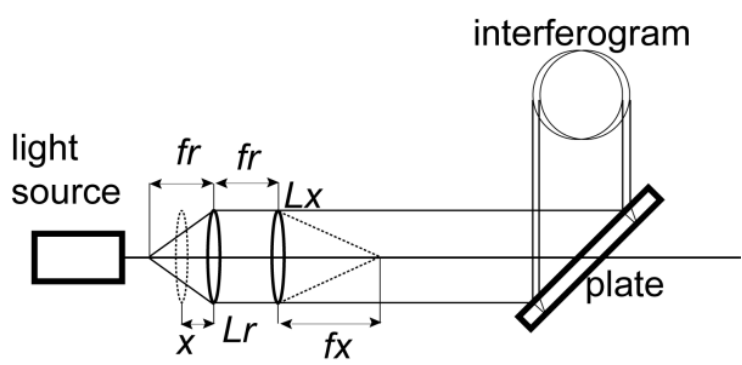

Fig. 15. Lateral shear interferometer proposed by GranadosAgustin et al [14].

system a laser beam is reflected by a series of mirrors (M2, M3 and M4) to pass through the tested lens and a collimating lens L3. When light is collimated light will focus on to the pinhole forming an interferogram that allows power calculation according to:

$$
P=\frac{M^{2} \lambda}{x S} \text {. }
$$

where $P$ is lens power, $x$ is fringe distance, $M$ is system amplification and $S$ is lateral displacement.

Recently Granados-Agustin et al [14] proposed a lensometer based in lateral shearing. The system is basically a He-Ne laser and a plane parallel plate, both located in the focal points of a reference lens as shown in Fig. 15.

The first step is to obtain an interferogram in absence of the ophthalmic lens to be tested. Once the reference interferogram has been observed the ophthalmic lens is included into the system, interferogram fringes rotate as a result. The reference lens is then moved along the optical axis a distance $x$ until the original interferogram gets visible again. The focal length $(x)$ of the 
ophthalmic lens is related to $x$ by means of the Newton's equation:

$$
x x^{\prime}=-f_{r}^{2} .
$$

The previous system is able to measure lenses in the range of $0.5 \mathrm{D}$ and $8 \mathrm{D}$ presenting an error less than $5 \%$.

\section{4.c. Point diffraction interferometer}

This kind of interferometer is commonly used to analyze wavefronts. Basically has a thin film with a pinhole in the center to diffract light when illuminated. The resulting wave front is spherical and serves as reference to perform optical measurements. The lens is placed in collimated light and a semitransparent membrane containing a pinhole is placed near the focus of the lens. The membrane transmits the test wave containing aberrations produced by the lens. The pinhole diffracts a spherical reference wave front. The two wave fronts interfere, and the resulting interference fringe pattern can be recorded and analyzed to reveal aberrations in the optical system [15].

Acosta et al [16] proposed an alteration in the point diffraction interferometer; they increased pinhole size to a diameter bigger than the Airy

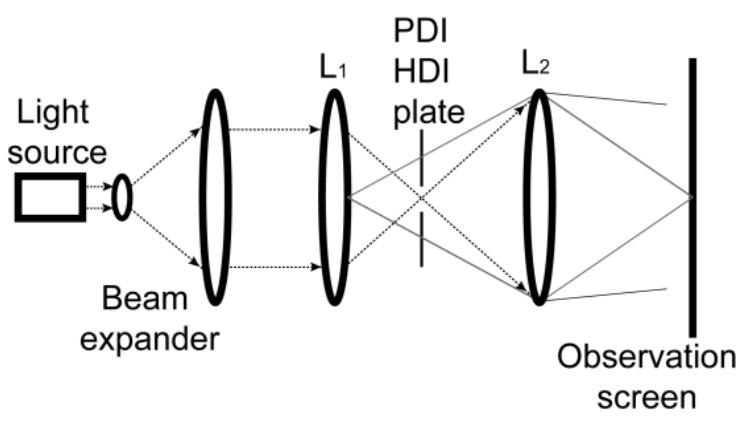

Fig. 16. Point and hole diffraction interferometer.

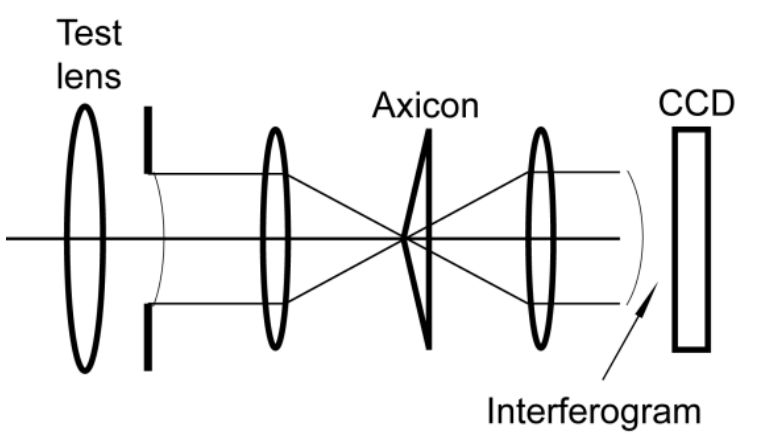

Fig. 17. Interferometer with an axicon. disk (see Fig. 16). The hole diffraction interferometer uses collimated light of a He- $\mathrm{Ne}$ laser. The lens to be tested placed in front of the system causes light to converge near the thin film containing the hole. Finally an interference pattern is observed and is directly related to sphere, cylinder and axis of the tested lens.

Koliopoulos et al [17] applied the point diffraction interferometer to test lenses, but they use an infrared light source. The infrared PDI is insensitive to vibrations but requires an expensive pyroelectric vidicon to image the interference fringes.

Vohnsen et al [18] developed a system similar to a PDI but using an axicon instead of the pinhole plate. The apex of the axicon acts like scattering center creating an interferometric reference wave. The axicon is rotationally symmetric and as a result the interferogram is a light ring that impinges on the CCD as shown in Fig. 17. Obviously in the presence of an ophthalmic lens the aberrations change the interferogram. The error in the system is approximately $2 \%$ for a lens of $1 \mathrm{D}$.

\section{4.d. Newton interferometer}

Newton rings are formed when two surfaces in contact are illuminated with a monochromatic light source. Between the two surfaces there is a gap occupied by air, this gap has variable thickness and produces a series of rings. This is because the test beam has no phase change and the optical path difference is zero so the two waves destructively interfere [19].

Newton rings allow calculating radii of curvature since there is a relation between the increment in rings radii and surface radii.

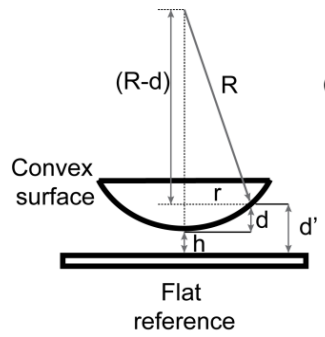

(a)

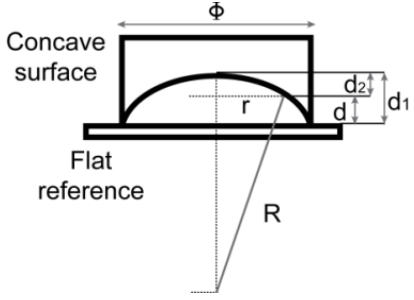

(b)
Fig. 18. Newton rings interferometer: (a) Convex surface, (b) Concave surface. 
Illueca et al [20] proposed an interferometric method using Newton rings. Through analysis of images 18(a) and 18(b) they obtain the formulas needed to calculate radii of curvature. In the case of a convex surface:

$$
r^{2}=m \lambda R
$$

In the case of a concave surface:

$$
r^{2}=\left(\frac{\Phi}{2}\right)^{2}-m \lambda R
$$

where $m$ is the fringe order, $\lambda$ is the wavelength and the other parameters are shown in the corresponding figures.

These equations are used along with the experimental setup of Fig. 19.

The system consists of a collimated $\mathrm{He}-\mathrm{Ne}$ laser source, followed by a filter and a diaphragm that attenuate and restrain light. Also the system uses a beam splitter to reflect the rays of light on to the lens holder containing the reference surface and the lens to be tested. Finally there is an observation and measuring system.

The authors measured spherical lenses (biconvex and meniscus) obtaining an error of $0.12 \mathrm{D}$ in power measurements. In the case of astigmatic lenses the error was approximately $0.7 \%$. And finally they measured progressive power lenses varying from $1 \mathrm{D}$ to $3 \mathrm{D}$ but the interference patterns are too complicated so 4 different points must be isolated and measured.

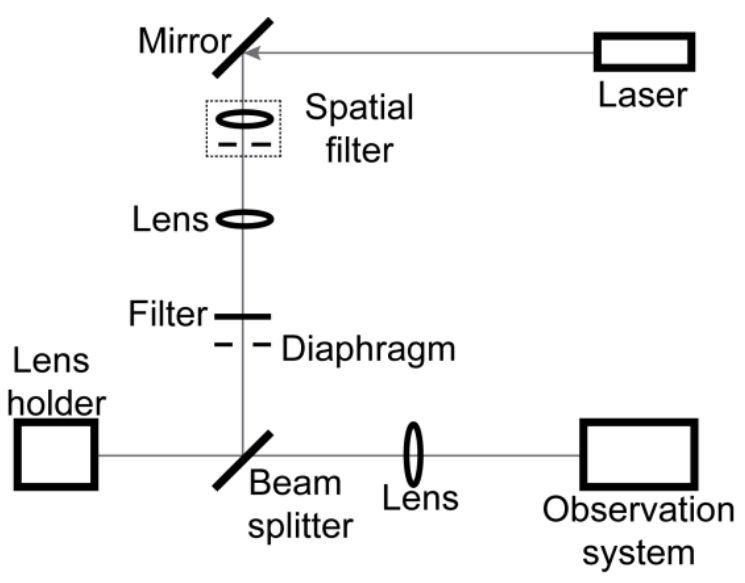

Fig. 19. Newton rings interferometer proposed by Illueca et al [20].

\section{4.e. Twyman-Green interferometer}

In this system, interference is produced by a light source collimated with a lens L1, a beam splitter divides the light and gets reflected by two perpendicular mirrors only to return to the beam splitter. This process forms two interferometric patterns, one going back to the observation system and another that returns to the light source (see Fig. 20). These patterns will be complementary to each other and contain information to obtain spherical aberrations and astigmatism of ophthalmic lenses, asymmetric aberrations such as coma won't appear [21].

To perform a measurement the system is adjusted to show no fringes, then by introducing the test lens in front of the light beam fringes will appear due to aberrations [12].

Pateras [22] uses a modification of a TwymanGreen interferometer to measure dioptric power in ophthalmic lenses. This system has the

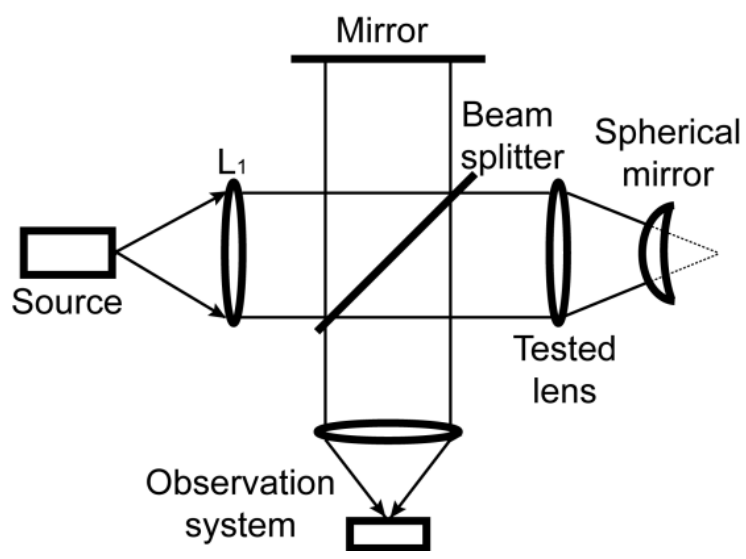

Fig. 20. Twyman- Green interferometer.

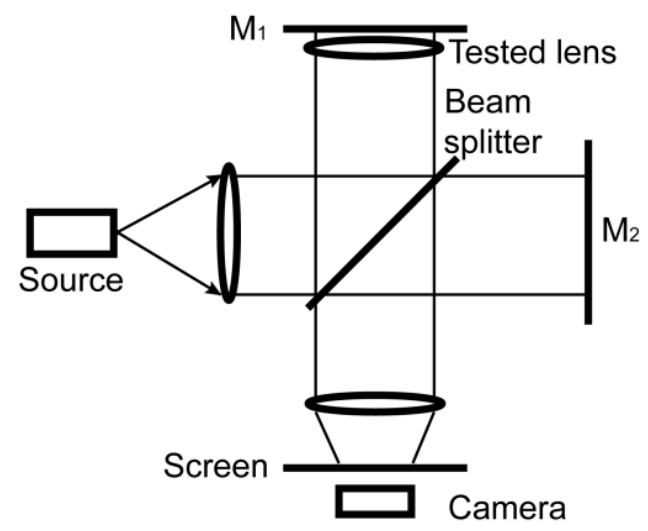

Fig. 21. Twyman- Green interferometer proposed by Pateras [22]. 
disadvantage of measuring only absolute power and is not capable of determining if positive or negative lens is. On this system M1 and M2 are perpendicular mirrors and the lens to be tested is placed in front of M1 (see Fig. 21).

\section{4.f. Talbot interferometry}

The Talbot effect consists in the formation of autoimages of a grating when this is illuminated with collimated light.

The Talbot image of a grating $\left(G_{1}\right)$ illuminated with a laser beam is formed at a distance:

$$
Z_{k}=\frac{k p^{2}}{\lambda}
$$

where $k$ is an integer corresponding to the number of the Talbot image, $p$ is the period of the grating, and $\lambda$ is the laser's wavelength.

If the illuminating wavefront is spherical or distorted, the fringes in the autoimage won't be straight. These phenomena can be seen from two different but equivalent points of view. The interferometric interpretation where the wavefront is diffracted by the grating and a lateral shear interferogram is formed. Or from the geometric point of view that will be treated later and is known as moiré deflectometry [12].

In Talbot interferometry the first ruling forms an autoimage in a multiple of the Rayleigh distance $\left(Z_{k}\right)$ and in this position a second ruling is placed. The superposition of the autoimage and the second ruling forms a moiré pattern and this allows to measure characteristics of the test object by introducing it into the system.

Nakano and Murata [23] use Talbot effect and Moiré to measure focal length. They place a ruling $G_{1}$ on the image plane a second ruling $G_{2}$ takes place, equal to $\mathrm{G}_{1}$ but rotated a $\theta$ angle. The superposition of both images will produce a fringe pattern. When $\theta \neq 0$ :

$$
f=\frac{Z_{k}}{\sin \theta \tan \alpha_{k}+\cos \theta-1}
$$

and if $\theta=0$ :

$$
f=\frac{k_{p} W}{\lambda}
$$

where $\alpha_{k}$ is the inclination angle of Moiré fringes and $W$ is the period of moiré fringes. The setup is shown in Fig. 22.

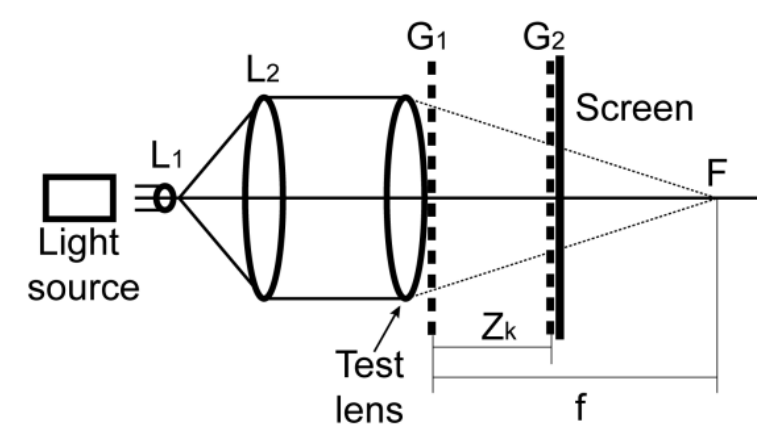

Fig. 22 Talbot interferometry proposed by Nakano and Murata [23].

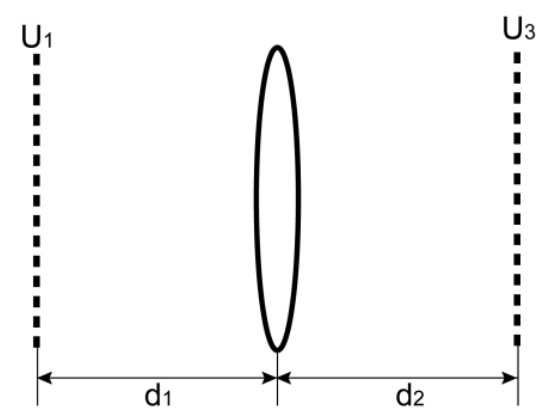

Fig. 23. Setup proposed by Bernardo and Soares [24].

When an ophthalmic lens is introduced in the system $\mathrm{G}_{1}$ stays fixed and $\mathrm{G}_{2}$ moves along the $\mathrm{z}$ axis in order to find the best contrast in the moiré fringes. The error obtained with this method is $2 \%$.

Bernardo and Soares [24] proposed to measure real focal length and demonstrated the advantages. They used a transparency of concentric squares instead of the gratings. The system consists of a transparency $\mathrm{U}_{1}$ at a distance $d_{1}$ from a lens that produces an amplitude distribution $\mathrm{U}_{3}$ at a distance $d_{2}$ from the lens as shown in Fig. 23.

If a second transparency $\mathrm{U}_{2}$ is located in $d_{2}$, Moiré fringes will result, where $\mathrm{F}$ is the inverse of the number of line pairs from $U_{1}$ contained in the spatial period of the pattern. To measure focal length they use the equation:

$$
f=d_{2} \frac{1+F_{2}}{2+F_{2}}
$$

where $F_{2}$ is the $F$ value when $d_{2}>f>0$. In the previous formula $d_{2}$ is difficult to obtain so the authors measure at two different distances and using the increment between them apply the next equation: 


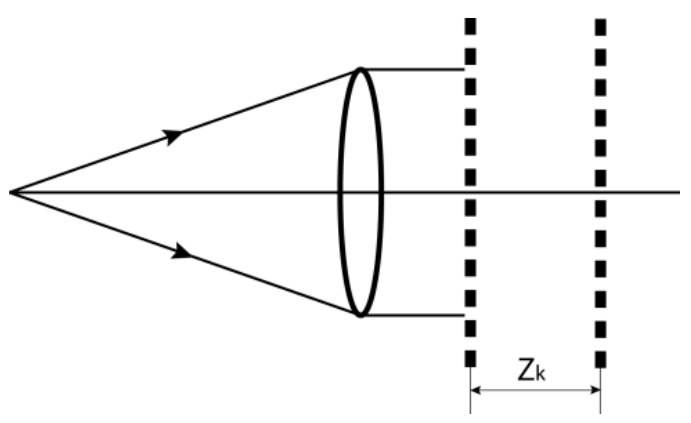

Fig. 24. Setup proposed by Bhattacharya and Aggarwal [25].

$$
f=\frac{\left(1+F_{2}^{\prime}\right)\left(1+F_{2}^{\prime \prime}\right)}{{F^{\prime}}_{2}-{F^{\prime \prime}}_{2}}\left(d_{2}^{\prime}-d^{\prime \prime}{ }_{2}\right) .
$$

The maximum error in focal length is $1 \%$.

Bhattacharya and Aggarwal [25] applied Talbot interferometry to measure focal length. Using two Ronchi rulings illuminated with monochromatic light Moiré fringes are formed. If the lens in the system presented in Fig. 24 is defocused the plane wavefront will become spherical and the image of the rulings is magnified (M):

$$
M=1+\frac{Z_{k}}{Z_{w}}
$$

where $Z_{w}$ is the curvature from the spherical wavefront and is related to the lens defocus.

To calculate focal length they used measures at two different distances $\left(x_{1}\right.$ and $\left.x_{2}\right)$ with the formula:

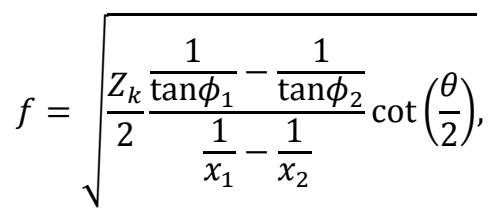

where $\phi$ is the resultant angle of putting $g_{2}$ in to the image of $\mathrm{G}_{1} . \theta$ is the angle of rotation of $\mathrm{G}_{2}$ respect to $G_{1}$. The error was approximately $2 \%$.

Sriram et al [26] used the setup shown in Fig. 25 to calculate focal length. The method is based in the fact that if a collimating beam impinges on the lens to be tested images of $\mathrm{G}_{1}$ will be formed at the left and right of the lens. The image at a distance $2 f$ has the same frequency than $G_{1} \cdot G_{2}$ moves to the position $\mathrm{G}_{2}^{\prime}$ and a field free of fringes is observed. The focal length is equivalent to the displacement of $\mathrm{G}_{2}$, the system

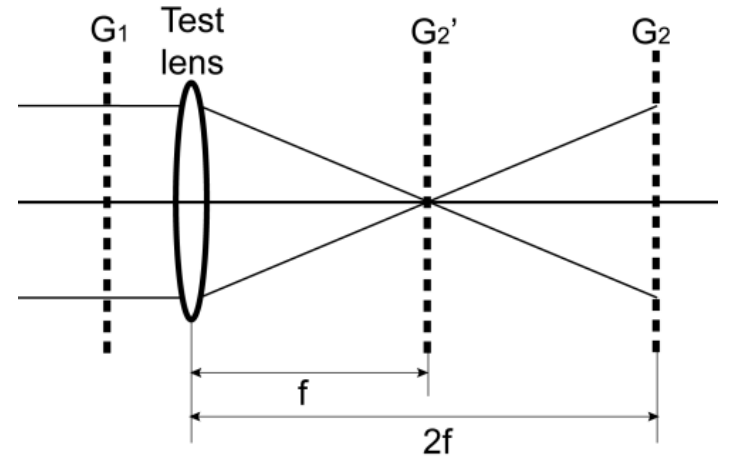

Fig. 25. Interferometer used by Sriram et al [26].

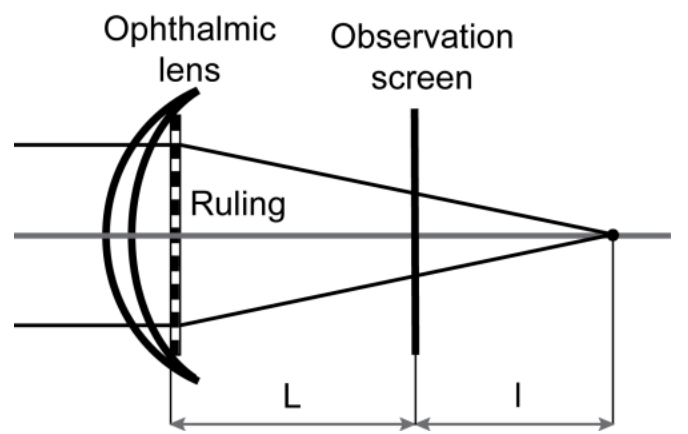

Fig. 26. Talbot interferometry.

is simple but no convenient for focal lengths over one meter.

Malacara-Doblado [27], proposed a method using Talbot interferometry. In this method a collimated light beam enters the lens to be tested, the light emerging from the lens is either convergent or divergent. A magnified autoimage of the ruling is seen at an observation screen (Fig. 26), the magnification allows measuring the curvature of the wavefront.

The observation distance is known and the spatial frequency of the projected fringe pattern is measured to calculate vertex focal length. Malacara-Doblado propose to measure fringe spatial frequency with the moiré fringes formed by the projected fringe pattern with a second ruling forming a small angle with respect to the first ruling.

The vertex power $P$ of a lens is the inverse of its back focal length $l$ in meters:

$$
P=\frac{1000}{l} \text {. }
$$


The period of the Ronchi ruling is $d$ and the period $d s$ on the screen of the observed periodic image is:

$$
d s=d \frac{l-L}{l} .
$$

Hence, the vertex power is:

$$
P=\frac{1000}{L}\left(1-\frac{d s}{d}\right) \text {. }
$$

\section{4.g. Moiré deflectometry}

Moiré deflectometry is a method based in the moiré and Talbot effects; it has been used to mapping ray deflections [28].

The geometrical approach to understand Talbot effect is called moiré deflectometry and occurs when a ruling is illuminated and the formed image is considered a shadow of the lines in the grating and the interference effects is seen as noise.

The basic deflectometer has a collimated beam and two Ronchi rulings. The shadow of $\mathrm{G}_{1}$ is projected over $\mathrm{G}_{2}$ as shown in Fig. 27.

In the observation screen a moiré pattern will be formed where the fringes are the solution to a series of equations. Each ruling is represented by:

$$
y=k p,
$$

where $k=0, \pm 1, \pm 2, \ldots$, and $p$ is the pitch.

If the rulings are rotated over the $y z$ plane an angle $\theta$, equations are:

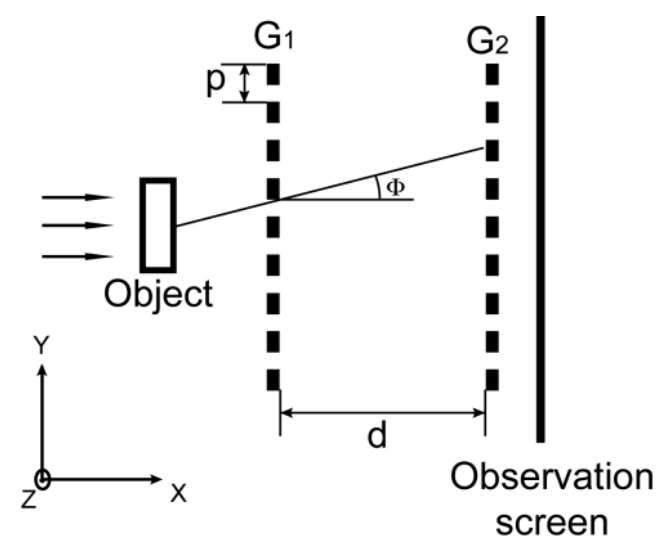

Fig. 27. Moiré deflectometer.

$$
\begin{gathered}
y \cos \left(\frac{\theta}{2}\right)=z \sin \left(\frac{\theta}{2}\right)+k p, \\
y \cos \left(\frac{\theta}{2}\right)=-z \sin \left(\frac{\theta}{2}\right)+k p .
\end{gathered}
$$

If the shadow of $\mathrm{G}_{1}$ over $\mathrm{G}_{2}$ is perfect, then:

$$
z=\frac{(m-k) p}{2 \sin \left(\frac{\theta}{2}\right)}=\frac{(m-k) p}{\theta}=\frac{l p}{\theta} .
$$

The ray deflection $\Phi$ changes the shadow produced by $\mathrm{G}_{1}$ an amount of $\Phi_{d}$ on $x$ direction, thus affecting the Moiré pattern. The deflection angle can be calculated using the fringe shift $z^{\prime}, \theta$ and $d$.

$$
\Phi(z, y)=\frac{z^{\prime} \theta}{d} .
$$

The previous equations are used in the finite configuration of the Moiré deflectometer. For infinite configuration $\theta=0$ and Eq. (23) changes to:

$$
\Phi(z, y)=\frac{l p}{d} .
$$

Diffraction is a limitation to this method accuracy.

To measure ophthalmic lenses is considered that the rotation of the fringes in a moiré pattern is directly related to the local focal length of a lens.

In Fig. 28 a lens under test $\mathrm{L}$ is placed before the gratings, the distortion of the Moiré deflectogram is proportional to the angular deflection of the incident rays passing through the lens.

The deflectograms allow calculating local fringe direction with intensity-based procedures [29].

Glatt and Kafri [30] used Moiré deflectometry (Fig. 29) to analyze lenses of focal lengths in the range of less than a meter to more than 5 meters. To apply their method they separate lenses in 3 regions: long focal length (over $5 \mathrm{~m}$ ), short focal length (below $1 \mathrm{~m}$ ) and an intermediate region. In the long focal length region the basic moiré deflectometer can be used and the focal length can be obtained with the formula:

$$
f \cong \frac{d}{\theta \tan \alpha}
$$




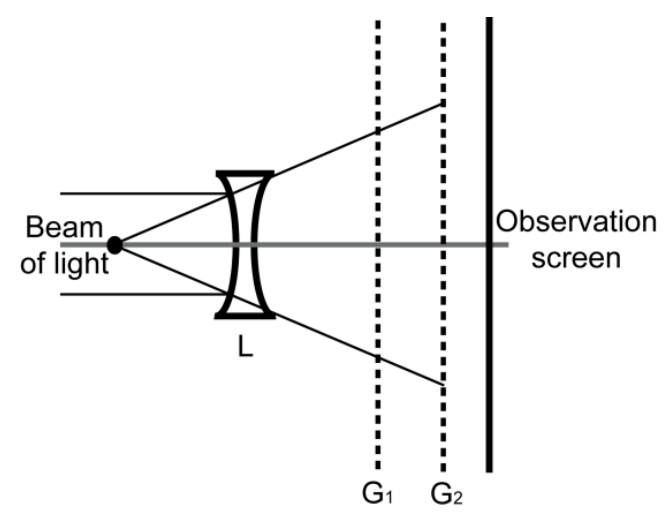

Fig. 28. Moiré deflectometer.

where $f$ is the focal length, $d$ is the distance between gratings, $\theta$ is the angle of intersection of the line direction and $\tan \alpha=h / r, h$ is the fringe shift and $r$ is the radial distance from the optical axis.

In the case of the intermediate region both gratings have parallel lines $(\theta=0)$. The converging lens will produce a beat pattern as a result of the mismatch between the image of the first grating and the second grating. The focal length can be calculated with:

$$
f=d \frac{p^{\prime}}{p}+l,
$$

where $f$ is focal length, $d$ is the distance between gratings, $p$ is the pitch of the gratings, and $p^{\prime}$ is the pitch of the beat pattern.

For short focal length region a corrective element in series with the tested lens must be introduced in order to produce a collimated beam. A mirror or a known focal length lens is suitable. The formula to calculate focal length in this case is:

$$
f=R-r,
$$

Keren et al [31] improved the previous system by proposing a universal method to test lenses regardless of the region. The method is based in the beat pattern observed by using the infinite mode of moiré fringes.

The authors claim that the aperture size a, number of fringes $N$, pitch $p$ and $\Delta$ (distance between gratings) are sufficient to calculate focal length according to the formula:

$$
f=\frac{a \Delta}{p N} .
$$

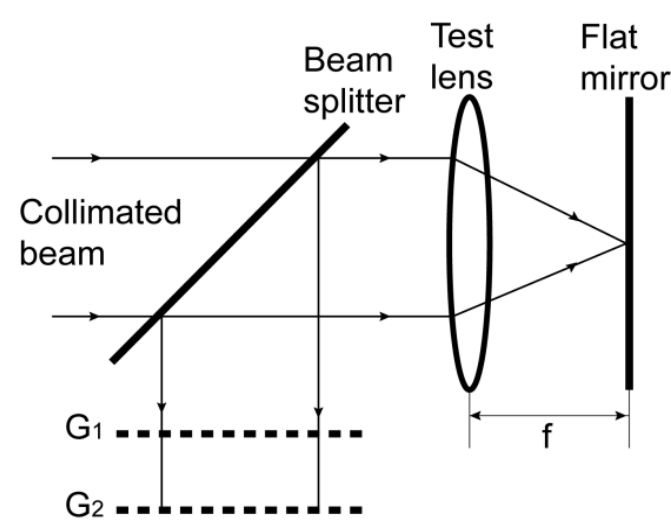

Fig. 29. Moiré deflectometer fom Glatt and Kafri [30] for short focal length.

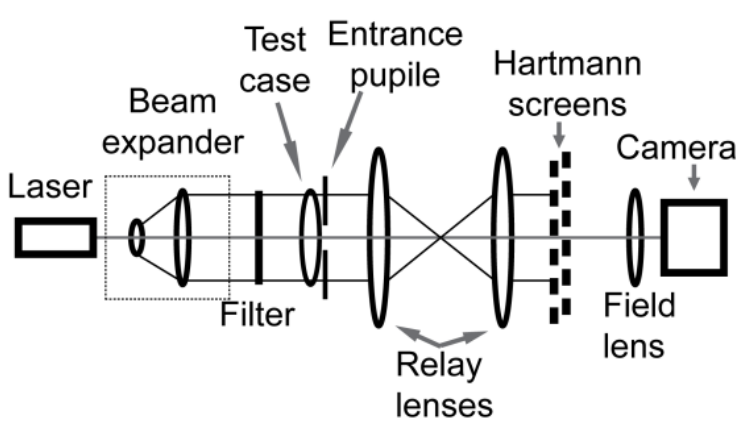

Fig. 30. Hartmann Moiré device.

The method is based on fringe counting and was demonstrated with lenses of $50 \mathrm{~cm}$ with satisfactory results.

Vargas et al [32] use Moiré deflectometry to calculate lens power as perceived by the user. The method uses a camera acting as pinhole located at the point where the center of rotation of the eye should be respect to the lens.

Canabal et al [33] developed and applied and automatic algorithm to extract the local fringe direction from deflectograms of ophthalmic lenses obtained with moiré deflectometry.

Wei et al [34] proposed a device using a collimated laser beam passing through a circular aperture and two Hartmann screens rotated between each other (Fig. 30). Light and both screens produce Moiré deflectograms; such patterns allow the calculation of cylindrical and spherical power. 


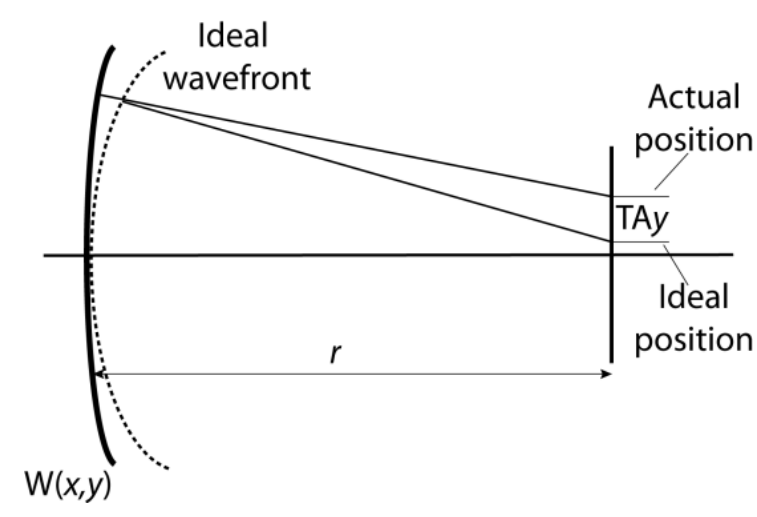

Fig. 31. Transverse aberration.

\section{4.h. Hartmann test}

The Hartmann test uses a perforated screen or disk; these holes are regularly spaced over the surface of the screen.

The disk is placed before the lens to be tested and a beam of light illuminates the optical system to obtain the transversal aberrations.

Wavefront deformations $W(x, y)$ are calculated from transversal aberrations TAX and TAy:

$$
\begin{aligned}
& \frac{\partial W(x, y)}{\partial x}=-\frac{a T A x(x, y)}{r}, \\
& \frac{\partial W(x, y)}{\partial y}=-\frac{a T A y(x, y)}{r},
\end{aligned}
$$

where $r$ is the distance between the pupil and the wavefront.

Hartmann patterns are spot diagrams and there are several methods to retrieve the wavefront aberrations, such as: trapezoidal integration, Southwell integration algorithm, polynomial fitting and other methods.

There are Hartmann test variations where only 4 holes are used. Also there is a well known variation to this test named Hartmann-Shack that uses a lenticular screen. This screen originally was a set of two layers of cylindrical lenses, now consists of an array of lenses in plastic, glass or silica (Fig. 32).

If a flat wavefront impinges on the sensor, light will be focused near its optical axis and if is aberrated a spot displacement will occur. HS sensors can easily detect and measure any power, and tell if negative or positive is. Also

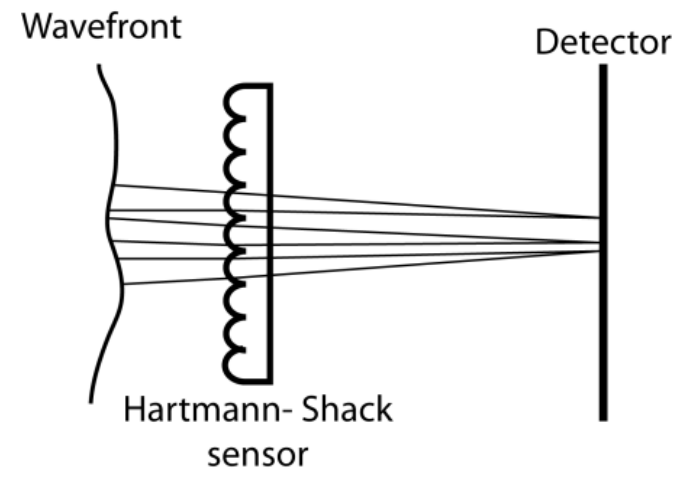

Fig. 32. Hartmann-Shack sensor.

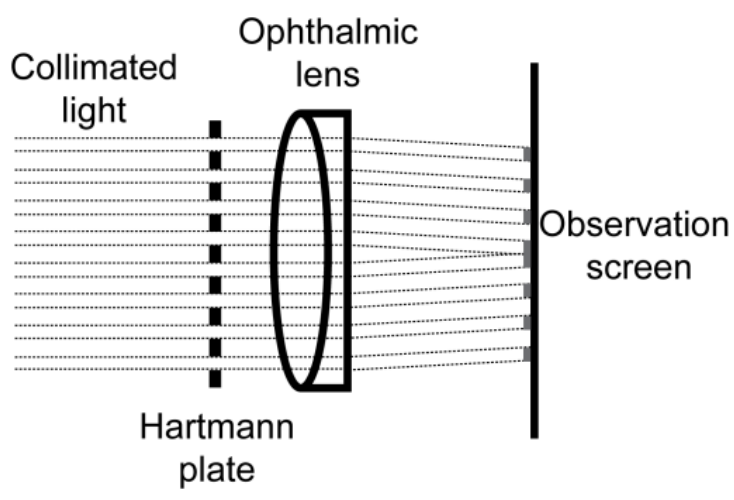

Fig. 33. Hartmann test.

since every spot is individually focused by each lenslet, the spot in the detector has more energy than with classic Hartmann.

Salas-Peimbert et al [35] presented a Hartmann test data interpretation to evaluate ophthalmic lenses using a collimated beam of light to illuminate a Hartmann plate. The light emerging through the holes in the plate hits the observation screen forming a Hartmann pattern (Fig. 33). Considering the spots as a set of test rays it is possible to use a ray tracing procedure to determinate the focal length of each spot (Fig. 34).

Analyzing a ray incident on the lens with a height $\mathrm{s}$ and refracted a distance TAs from its reference position $A$ to its test position $B$ on the observation screen. This refracted ray forms an angle $\alpha$ with the original ray trajectory forming two triangles with the same angle $\alpha$ : ABC and $A^{\prime} B^{\prime} C$. Considering that the tangent of both triangles is equal:

$$
\frac{f_{s}}{s}=\frac{r}{T A s} \Rightarrow f_{s}=\frac{s r}{T A s} .
$$




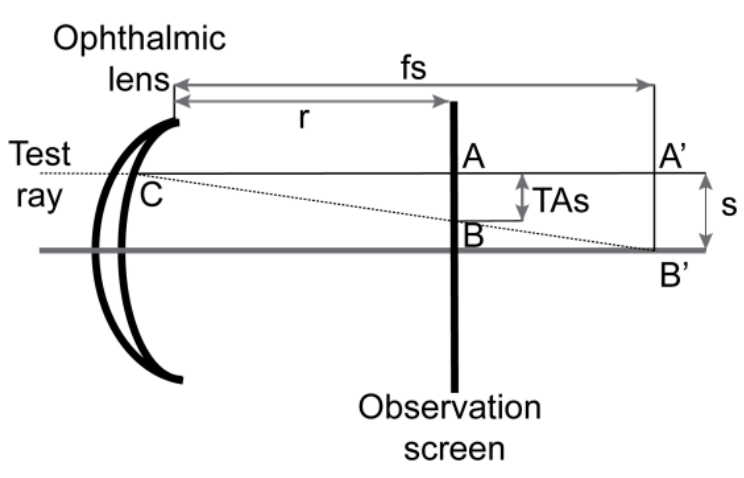

Fig. 34. Geometric analysis of Hartmann test.

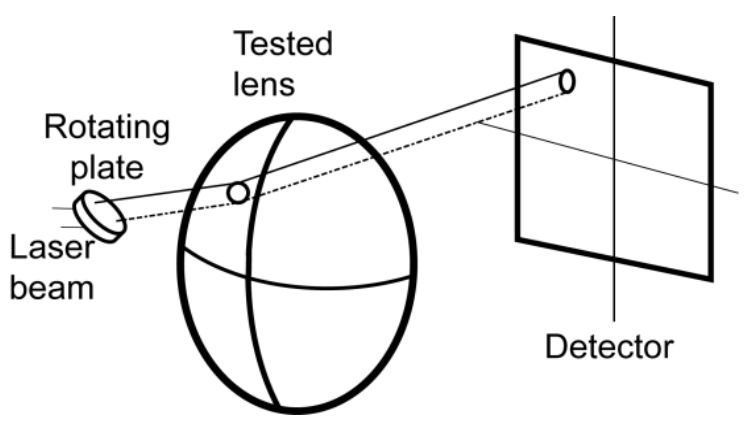

Fig. 35. Hartmann test proposed by Castellini et al [36].

The power in the orientation of the $\mathrm{S}$ axis is the inverse of $f$ :

$$
P_{s}=\frac{T A s}{s r} .
$$

Castellini et al [36] modified the Hartmann test using a circular scanning laser beam instead of the perforated plate. The beam impinges on a rotating plane parallel plate passes through the lens and falls in a position sensitive detector (Fig. 35). The coordinates allow calculating the wavefront by using Fourier series. The system is capable of analyzing all the surface of the lens by using a mechanical support that simulates real human behavior of the eye.

Chuanquing et al [37] proposed a system based in a Hartmann Shack sensor to retrieve the wavefront with Zernike polynomials. They used the apparatus to compare four types of progressive addition lenses. To perform the Hartmann test they mount the ophthalmic lens in a positioning stage that allows lens movement to analyze the whole surface of the lens.

Doyle [38] proposed a simplified Hartmann test where the lens to be tested is not necessarily

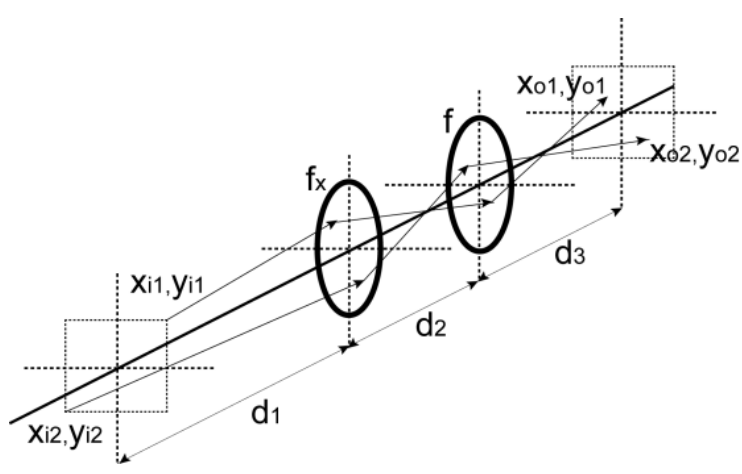

Fig. 36. Hartmann test proposed by Doyle [38].

aligned to the rest of the optic system. According to Fig. 36 two laser beams parallel and symmetric about the optical axis enter the system, pass through the lens to be tested (focal distance $f_{x}$ ) and a reference lens (known focal length $f$ ) and impinge on a position sensitive detector.

In the input plane the rays are located in $\left(x_{i 1}, y_{i 1}\right)$ and $\left(x_{i 2}, y_{i 2}\right)$, on the output plane the location is $\left(x_{o 1}, y_{01}\right)$. Being the beams parallel $x_{i 1}=-x_{i 2}=x_{i}$. The average of the output ray positions $X$ is represented with the formula:

$$
X=-x_{i} \frac{f}{f_{x}} .
$$

$X$ is obtained from the position detector and $f_{x}$ can be calculated.

Villegas and Artal [39] presented a Hartmann Shack sensor to measure optical characteristics of progressive power lenses and the eye separately or in combination. They measure PPL's in normal viewing conditions retrieving the wavefront aberrations with the Zernike polynomials.

Qin and Yu [40] use Hartmann test to measure focal length in progressive power lenses using 3 spots of the Hartmann pattern to perform the analysis.

\section{4.i. Ronchi test}

Originally this method was used to measure mirror aberrations. Consists in place a ruling near the center of curvature of the tested mirror, this way the ruling image superposes with the ruling itself, forming patterns dependent of the mirror aberrations. This test has two equivalent models: A geometric one that considers fringes as shadows from the ruling lines, and a physics 
one where the fringes are interpreted as shadows due to diffraction and interference.

According to Malacara [21] the Ronchi test measures the transverse aberration TA in a direct way. The geometrical model and formula are:

$$
\frac{\partial W}{\partial x} \cos \varphi-\frac{\partial W}{\partial y} \sin \varphi=-\frac{m d}{r},
$$

where $r$ is the distance from the tested surface to the ruling (Fig. 37).

In the physical model the ruling acts as a diffraction ruling producing a laterally sheared image (Fig. 38). So it can be seen as an interferometer. Both models end up in the same result.

Gonzalez et al [41] proposed a modification to Ronchi test; they placed a diffraction ruling near the focus of the tested lens forming interferograms at an observing screen. The interferograms have the information needed to measure the lens aberrations.

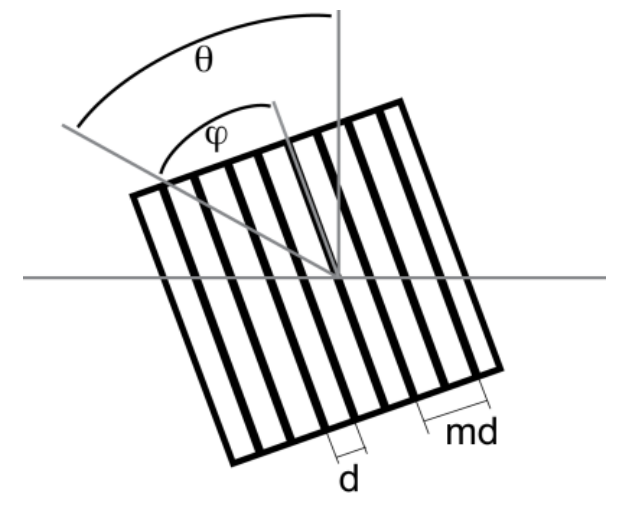

Fig. 37. Geometrical approach of the Ronchi test.

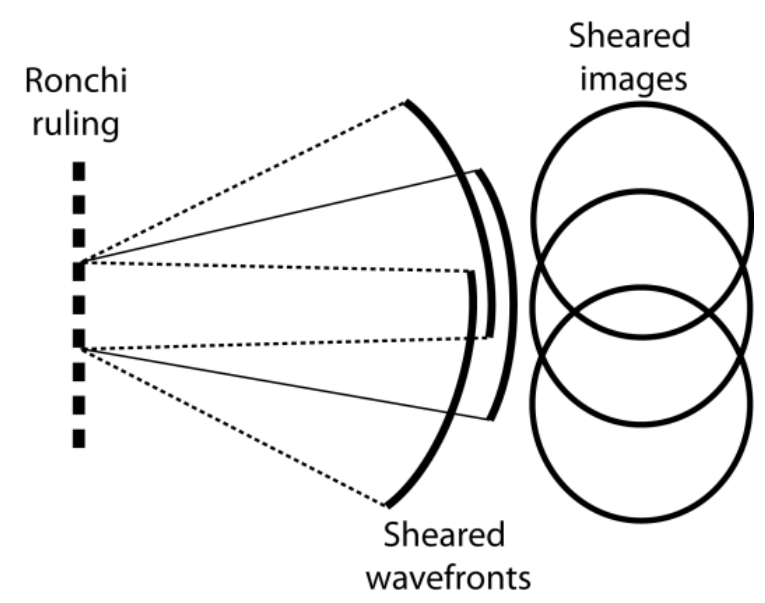

Fig. 38. Physical model of the ronchi test.
Carretero et al [42] applied Ronchi test to the measurement of intraocular lenses. They locate a grating near the image point of the intraocular lens, then the grating is moved until light emerging from the system produces interference fringes (Fig. 39). The focal length is equal to the distance between the lens and the ruling.

Buah et al [43] fabricated a simple lensometer based on Ronchi test. The system uses the environment light to illuminate two gratings $\left(\mathrm{G}_{1}\right.$ and $G_{2}$ ) mounted in an annular support at a distance $\left(x_{1}\right.$ and $\left.x_{2}\right)$ from the lens. From the two gratings a moiré pattern results where the fringes in the pattern are rotated an angle $\alpha$. The focal length of the lens is related to $\tan \alpha$ in the formula:

$$
\tan \alpha=-\frac{d}{\theta f}
$$

where $\theta$ is the angle of rotation of $\mathrm{G}_{2}$ respect to $\mathrm{G}_{1}$. The device must be calibrated in absence of the lens and the precision is of $0.25 \mathrm{~m}^{-1}$.

\section{4.j. Fringe reflection}

This method projects fringes over the lens to be measured and a camera located at a triangulation angle records the scattered light (Fig. 40). Evaluated absolute phase identifies the

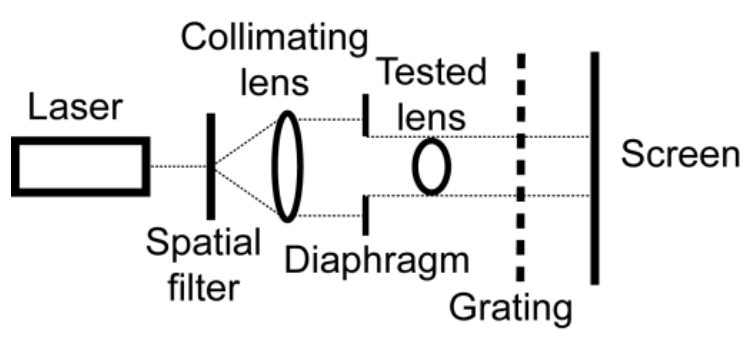

Fig. 39 Ronchi test proposed by Carretero et al [42].

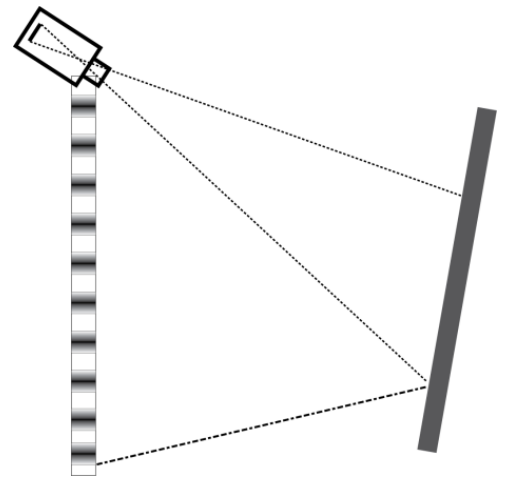

Fig. 40. Setup for fringe reflection proposed by Bothe et al [43]. 
location of the originating fringe, in consequence reflection angles for every camera pixel can be identified [44].

The setup can be a camera affixed to the border of the monitor, camera and object are tilted to meet the reflection conditions.

The system does not require stabilization, works in rough environments where interferometric methods can't.

\section{Conclusions}

Ophthalmic lenses are the most commonly used solution to visual affections such as myopia, hyperopia, presbyopia and astigmatism. However an ophthalmic lens has a series of optical characteristics relevant to visual acuity. Lensometers are the tool to measure such characteristics. Nowadays several methods and techniques are the base to develop ophthalmic equipment, but the opportunity of improvement is still latent.

The focimeter is a simple tool that allows focal length measurements but sacrifices accuracy since is user dependent nevertheless is the base for the classic American style lensometer. Basically is an optic system designed to see a focus image of a movable target, when the lens to be tested is introduced into the system the target image looks fuzzy, then the user moves the target until is finally clear again. The moved distance is related to the focal length of the measured lens. The interferometric techniques show great accuracy but the system and the data interpretation are more complex than in other methods. There are several interferometric methods such as lateral shear that uses collimated light impinging on a plane parallel plate to produce an interferogram, the plane parallel plate reflects light in both faces causing interference, introducing the ophthalmic lens will produce interference fringes to measure lens characteristics, is a simple system with an error minor to $5 \%$. The Ronchi test is a method able to measure ophthalmic lenses using a ruling near the image point of the lens and the moving it until fringes appear. Ronchi test has two different models, the physics one where is similar to a laterally sheared interferometer due to diffraction and the geometrical one where transversal aberrations are measured.

In a similar case it can also be found moiré deflectometry and Talbot interferometry, two equivalent methods that require a set of two gratings, where the shadow of the first one superposes with the second and forms a moiré pattern. Moiré deflectometry analyzes fringes as shadows from the projected lines of the grating; Talbot interferometry sees the fringes as an interferogram of laterally sheared wavefronts.

Twyman-Green is another interferometric method that uses two mirrors and a beam splitter to create interference fringes, by introducing the lens to be tested system conditions change and produce fringes with information about the lens aberrations. This method measures only absolute power and is not able to tell if the lens is negative or positive.

Newton ring is also an interferometric method where the contact between two surfaces produces a series of rings with the data to characterize a lens. The point diffraction interferometer and its variations: hole diffraction and axicon, use a film with a pinhole or an axicon illuminated to produce a reference spherical wavefront.

It is important to say that most interferometric methods are highly accurate with an error less than $2 \%$, but require controlled conditions, are susceptible to noise and vibrations, also some of the authors worked with a limited range of dioptric power of ophthalmic lenses.

In the case of Hartmann test is one of the most used methods to measure power in lenses and also most of the commercial equipment uses this technique. Requires a screen with holes to project a spot pattern on to a screen or detector, then when a lens is introduced into the system the pattern changes and transversal aberration can be measured.

\section{Acknowledgements}

The authors wish to acknowledge the financial support of the Dirección General de Educación Superior Tecnológica (DGEST), México. 